\title{
Prevalência e fatores associados à fragilidade em idosos institucionalizados das regiões Sudeste e Centro-Oeste do Brasil
}

\section{Prevalence and factors associated to frailty in institutionalized elderly of Southeastern and Middle- Western Brazil}

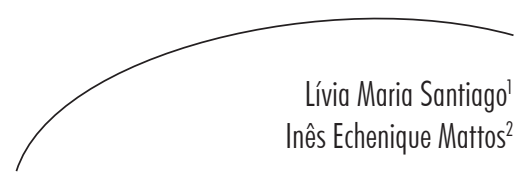

\section{Resumo}

Objetivos: Estimar a prevalência de fragilidade e identificar os fatores associados à fragilidade em indivíduos residentes em instituições de longa permanência para idosos das regiões Centro-Oeste e Sudeste do Brasil. Métodos: Estudo seccional, com 442 idosos institucionalizados de quatro municípios brasileiros. A fragilidade foi avaliada pela versão brasileira do Tilburg Frailty Indicator (TFI). Foram descritas as características da população e estimada a prevalência da fragilidade. Efetuou-se a descrição dos escores total e dos domínios do instrumento. Utilizou-se a regressão de Poisson para explorar as associações observadas, após ajuste para confundimento. Resultados: Grande parte da população era masculina (64,3\%), escolarizada $(74,1 \%)$ e a média de idade era de 75,0 (9,9) anos. A prevalência de fragilidade foi de 52,0\%. A média do escore total do TFI foi de $4,9(2,5)$ pontos e as médias dos escores dos domínios físico, social e psicológico corresponderam a 2,8 (1,7), 1,3 $(0,9)$ e $0,8(0,8)$, respectivamente. Mostraram-se associadas à fragilidade em idosos institucionalizados: idade elevada, analfabetismo, comorbidades e polifarmácia. No modelo multivariado, somente analfabetismo e comorbidades mantiveram sua significância estatística ( $\mathrm{RP}=1,28$ IC95\% 1,07-1,54 e RP=1,48 IC95\% 1,21-1,81). Conclusões: A prevalência de fragilidade foi maior do que a observada nos outros dois estudos que avaliaram idosos institucionalizados. As associações identificadas são corroboradas pela literatura científica. O TFI é um instrumento multidimensional baseado em um conceito integral da fragilidade e a versão brasileira se mostrou adequada para avaliar esta condição em idosos institucionalizados. Entretanto, é importante realizar estudos que avaliem sua utilização em idosos residentes na comunidade.

\footnotetext{
Departamento de Fonoaudiologia, Faculdade de Medicina. Universidade Federal do Rio de Janeiro. Rio de Janeiro, RJ, Brasil.

2 Departamento de Epidemiologia e Métodos Quantitativos, Escola Nacional de Saúde Pública. Fundação Oswaldo Cruz. Rio de Janeiro, RJ, Brasil.
}

Palavras-chave: Fragilidade. Prevalência. Determinantes. Idosos. Instituições de Longa Permanência para Idosos. 


\section{Abstract}

Aims: To estimate the prevalence of frailty and identify the associated factors in residents of homes for the aged of Southeast and Middle West regions of Brazil. Methods: Sectional study with 442 institutionalized elderly in four Brazilian municipalities. Frailty was assessed using the Brazilian version of the Tilburg Frailty Indicator (TFI). The characteristics of the study population were described and the prevalence of frailty was estimated. Means of the total score of TFI and of the scores of its domains were verified. Poisson's regression was applied to explore the observed associations, after adjustment for confounding. Results: The study population was predominantly male $(64.3 \%)$ and schooled $(74.1 \%)$ and mean age was 75.0 (9.9) years. Prevalence of frailty was $52.0 \%$. The mean of TFI's total score was 4.9 (2.5) points and the means of domains physical, psychological and social were, respectively, $2.8(1.7), 1.3(0.9)$ and 0.8 (0.8). The following variables were associated with frailty in institutionalized elders: advanced age, illiteracy, comorbidity and polipharmacy. In the multivariate model, only the associations with illiteracy and comorbidity maintained statistical significance $(\mathrm{PR}=1.28 \mathrm{CI} 95 \% 1.07-1.54$ and $\mathrm{PR}=1.48 \mathrm{CI} 95 \%$ 1.21-1.81). Conclusions: The prevalence of frailty was higher than the observed in two other studies that assessed this condition in institutionalized elders. The identified associations are corroborated by the scientific literature. The TFI is a multidimensional instrument based on an integral concept of frailty and the Brazilian version was adequate to evaluate this condition in institutionalized elders. However, it is important to conduct studies to assess its usefulness in elderly community residents.
Key words: Frailty. Prevalence. Epidemiologic Factors. Elderly. Homes for the Elderly.

\section{INTRODUÇ̃̃O}

Entre as condições de saúdeno envelhecimento, que vem ganhando destaque mundial, encontrase a fragilidade, ${ }^{1,2}$ considerada como uma resultante da diminuição da reserva fisiológica e da capacidade de manutenção da homeostase em indivíduos idosos, tornando-os mais vulneráveis às situações de estresse ambiental. ${ }^{3}$ Tal condição tem sido associada a elevado risco de desfechos adversos de saúde, como hospitalização recorrente, incapacidade funcional, institucionalização e morte prematura. ${ }^{4-7}$

Desde o surgimento do termo, têm sido debatidas duas abordagens conceituais distintas que determinam o modo de avaliação da fragilidade: a unidimensional, que considera somente características físicas para sua identificação; ${ }^{4}$ e a multidimensional, que agrega outros domínios. ${ }^{8-10} \mathrm{O}$ conceito multidimensional da fragilidade é o que tem predominado atualmente $^{8,11}$ e entre os instrumentos de avaliação propostos dentro desta perspectiva, destaca-se o Tilburg Frailty Indicator (TFI).

O TFI é um instrumento desenvolvido na Holanda, constituído por 15 questões objetivas, distribuídas em três domínios (físico, psicológico e social), que pode ser aplicado por qualquer profissional de saúde, utilizando baixo recurso financeiro e de tempo. ${ }^{12}$ Recentemente, foi efetuado o processo de adaptação transcultural do TFI para a população idosa brasileira, cumprindose as etapas de avaliação das equivalências conceitual, de itens, semântica e operacional e o pré-teste da versão do instrumento, ${ }^{13}$ bem como a avaliação de suas propriedades psicométricas (confiabilidade e validade de constructo). ${ }^{14}$ Os resultados apontaram a versão brasileira do instrumento como uma ferramenta útil para avaliação da fragilidade em nosso país. 
Segundo a legislação brasileira, a família é a principal fonte de apoio e cuidado dos idosos, devendo responsabilizar-se pela sua participação na comunidade, defesa de sua dignidade e bemestar e garantia de seu direito à vida. ${ }^{15}$ Entretanto, os indivíduos em idade avançada, que apresentam comprometimento físico e/ou cognitivo expressivo e em situação de vulnerabilidade social gerada por renda insuficiente, conflitos ou ausência de familiares, podem ser encaminhados para instituições de longa permanência para idosos (ILPI). ${ }^{16}$

As ILPIs brasileiras, segundo a Portaria SEAS n ${ }^{\circ} 2.874 / 2000$, são uma modalidade de cuidado destinada à moradia coletiva de indivíduos de 60 anos de idade ou mais, com ou sem suporte familiar, vinculadas ou não à iniciativa governamental. ${ }^{17}$ Os residentes das ILPIs apresentam grande heterogeneidade em suas condições de saúde, visto que sua admissão pode ser determinada tanto por questões relacionadas à higidez, quanto por aquelas de cunho de assistência social.

Embora haja um interesse crescente pela saúde do idoso na literatura científica, constatase um número reduzido de investigações voltadas para os idosos institucionalizados. Em relação à fragilidade, só foram identificados três estudos que contemplam esse grupo populacional específico. ${ }^{18-20}$

Este estudo objetivou estimar a prevalência de fragilidade e identificar os fatores associados à fragilidade em indivíduos residentes em instituições de longa permanência para idosos das regiões Centro-Oeste e Sudeste do Brasil.

\section{MATERIAL E MÉTODOS}

Para o desenvolvimento do estudo, foram utilizados dados da pesquisa "Condições de saúde de idosos institucionalizados: uma proposta de avaliação de necessidades e utilização da Classificação Internacional de Funcionalidade, Incapacidade e Saúde para planejamento de ações de saúde", realizada no período de 2010 a 2011, com indivíduos de 60 anos de idade ou mais, residentes em ILPIs de quatro municípios brasileiros (Rio de Janeiro-RJ, Juiz de Fora-MG, Campo Grande-MS e Cuiabá-MT).

Para o estudo seccional, foram selecionados os 442 idosos institucionalizados que eram capazes de responder às questões autorreferidas integrantes do instrumento de coleta de dados da investigação. Foram excluídos os indivíduos que apresentavam condições como demência, distúrbios psiquiátricos, deficiência mental, sequela de acidente vascular celebral (AVC) com comprometimento da linguagem, cegueira e surdez.

A fragilidade, variável dependente do estudo, foi avaliada pela versão brasileira do instrumento Tilburg Frailty Indicator (TFI), validada para a população do país, apresentada no quadro $1 .{ }^{13}$ Originalmente, o TFI é composto por duas partes: a primeira, denominada Parte A, que trata dos determinantes da fragilidade; e a segunda, Parte $\mathrm{B}$, que corresponde à avaliação da condição propriamente dita. A Parte B foi o alvo da adaptação transcultural, sendo, portanto, objeto deste estudo. É composta por 15 questões objetivas, distribuídas nos domínios "físico", "psicológico" e "social", às quais é atribuída a pontuação "zero" ou "um". Os escores desses domínios variam, respectivamente, de 0-8, 0-4 e $0-3$. O escore final do instrumento pode variar entre zero e 15 pontos e, quanto mais elevado, maior o nível de fragilidade ou, alternativamente, escores $\geq 5$ pontos indicam que o indivíduo avaliado é frágil. ${ }^{12-14}$ Nesta investigação, considerou-se frágil o indivíduo que obteve pontuação igual ou superior a cinco no TFI. 
Quadro 1. Versão brasileira do Tilburg Frailty Indicator (TFI).

\begin{tabular}{|c|c|}
\hline \multicolumn{2}{|l|}{ Domínio físico } \\
\hline (1) Você se sente saudável? & ( ) $\operatorname{sim}$ ( ) não \\
\hline $\begin{array}{l}\text { (2) Você perdeu muito peso recentemente sem querer que isso } \\
\text { acontecesse? ( }>6 \mathrm{~kg} \text { nos últimos seis meses ou }>3 \mathrm{~kg} \text { no último mês). }\end{array}$ & ( ) $\operatorname{sim}$ ( ) não \\
\hline (3) No seu dia a dia a dificuldade de caminhar lhe traz problemas? & ( ) $\operatorname{sim}$ ( ) não \\
\hline $\begin{array}{l}\text { (4) No seu dia a dia a dificuldade em manter o equilíbrio lhe traz } \\
\text { problemas? }\end{array}$ & ( ) $\operatorname{sim}$ ( ) não \\
\hline (5) No seu dia a dia a audição ruim lhe causa problemas? & ( ) $\operatorname{sim}$ ( ) não \\
\hline (6) No seu dia a dia a visão ruim lhe causa problemas? & ( ) $\operatorname{sim}$ ( ) não \\
\hline (7) No seu dia a dia a fraqueza nas mãos lhe causa problemas? & ( ) $\operatorname{sim}$ ( ) não \\
\hline (8) No seu dia a dia o cansaço lhe causa problemas? & ( ) $\operatorname{sim}$ ( ) não \\
\hline \multicolumn{2}{|l|}{ Domínio psicológico } \\
\hline (9) Você tem problemas de memória? & ( ) sim ( ) às vezes ( ) não \\
\hline (10) Você se sentiu triste no último mês? & ( ) sim ( ) às vezes ( ) não \\
\hline (11) Você se sentiu nervoso ou ansioso no último mês? & ( ) sim ( ) às vezes ( ) não \\
\hline (12) Você enfrenta bem os problemas? & ( ) $\operatorname{sim}$ ( ) não \\
\hline \multicolumn{2}{|l|}{ Domínio social } \\
\hline (13) Você mora sozinho? & ( ) $\operatorname{sim}$ ( ) não \\
\hline (14) Você sente falta de ter pessoas ao seu lado? & ( ) sim ( ) às vezes ( ) não \\
\hline (15) Você tem apoio suficiente de outras pessoas? & ( ) $\operatorname{sim}$ ( ) não \\
\hline
\end{tabular}

Questões 1, 12 e 15: $\operatorname{sim}=0$ e não=1; questões $2-8$ e 13: $\operatorname{sim}=1$ e não=0; questão 9: $\operatorname{sim}=1$ e não/às vezes $=0$; questões 10,11 e 14 : $\operatorname{sim} /$ às vezes $=1$ e não $=0$.

Foram avaliados como possíveis fatores associados à fragilidade, covariáveis sociodemográficas (localização da ILPI, sexo, faixa etária, escolaridade e status conjugal), relacionadas à saúde (comorbidades e de medicamentos utilizados), aos hábitos de vida (tabagismo e consumo de álcool) e aos aspectos socioculturais (participação em atividades de lazer e religião). Para a análise múltipla, a variável idade foi estratificada em duas categorias.

Efetuou-se a análise descritiva da população de estudo por meio de medidas de tendência central para as variáveis contínuas e de distribuição de frequência para as variáveis categóricas. Foi estimada a prevalência de fragilidade, com base no ponto de corte preconizado para o
TFI. Foram também calculadas as médias do escore total do TFI e dos escores parciais de cada domínio que compõe o instrumento e realizada a distribuição dos casos de fragilidade identificados, considerando os domínios individuais e os conjuntos de domínios afetados.

Foram calculadas razões de prevalência (RP) para investigar a existência de associações entre as variáveis independentes e a fragilidade. As razões de prevalências ajustadas foram obtidas por meio da análise múltipla de regressão de Poisson com variância robusta, considerando as variáveis independentes que estiveram mais fortemente associadas com a fragilidade na análise bivariada (nível de significância igual ou menor que 0,20 ). Para todas as análises, considerou-se um nível de 
significância de 0,05 . O software STATA SE 10 (StataCorp LP) foi utilizado para realização das análises estatísticas.

Este estudo foi aprovado pelo Comitê de Ética em Pesquisa da Escola Nacional de Saúde Pública / Fundação Oswaldo Cruz, sob o n ${ }^{\circ}$ CAAE: 0199.0.031.000-11. Os termos de consentimento livre e esclarecido (TCLE) foram assinados pelos indivíduos ou, em caso de incapacidade de assinar seus próprios nomes, foram assinados por seus representantes legais na instituição.

\section{RESULTADOS}

A média de idade da população de estudo foi de 75,0 ( $\pm 9,9)$ anos, com mediana de 74,0 anos. Grande parte dos indivíduos era do sexo masculino (68,1\%), alfabetizados $(74,1 \%)$ e sem companheiro (solteiros ou viúvos) (75,8\%). Quanto às características de saúde e estilo de vida, 52,3\% dos idosos apresentavam duas ou mais morbidades, 29,8\% utilizavam cinco ou mais medicamentos, $65,9 \%$ referiram tabagismo (atual ou anterior) e 66,5\% referiram hábito de consumir bebidas alcoólicas (atual ou anterior). No que se refere aos aspectos socioculturais, $93,3 \%$ dos sujeitos referiram ter religião (tabela 1).

A prevalência de fragilidade estimada para a amostra, segundo o ponto de corte proposto para o TFI, foi de $52 \%$. A média do escore total deste instrumento na população avaliada foi de $4,9(2,5)$ pontos. No domínio físico, o escore médio correspondeu a $2,8(1,7)$, para o domínio psicológico a $1,3(0,9)$ e para o domínio social a $0,8(0,8)$.

Na figura 1, pode-se observar a relação entre os domínios na determinação da fragilidade. Os valores apresentados correspondem à prevalência de fragilidade, caso o instrumento considerasse os domínios individualmente e/ou suas interseções com outro(s) domínio(s). Como exemplo, se o TFI fosse composto apenas de itens do domínio físico, somente três $(0,7 \%)$ idosos seriam considerados frágeis; se acrescentássemos o domínio social, mais $13(2,9 \%)$ indivíduos seriam identificados; entretanto, quando se levou em conta os três domínios que compõem o instrumento, foi possível identificar 230 idosos frágeis.

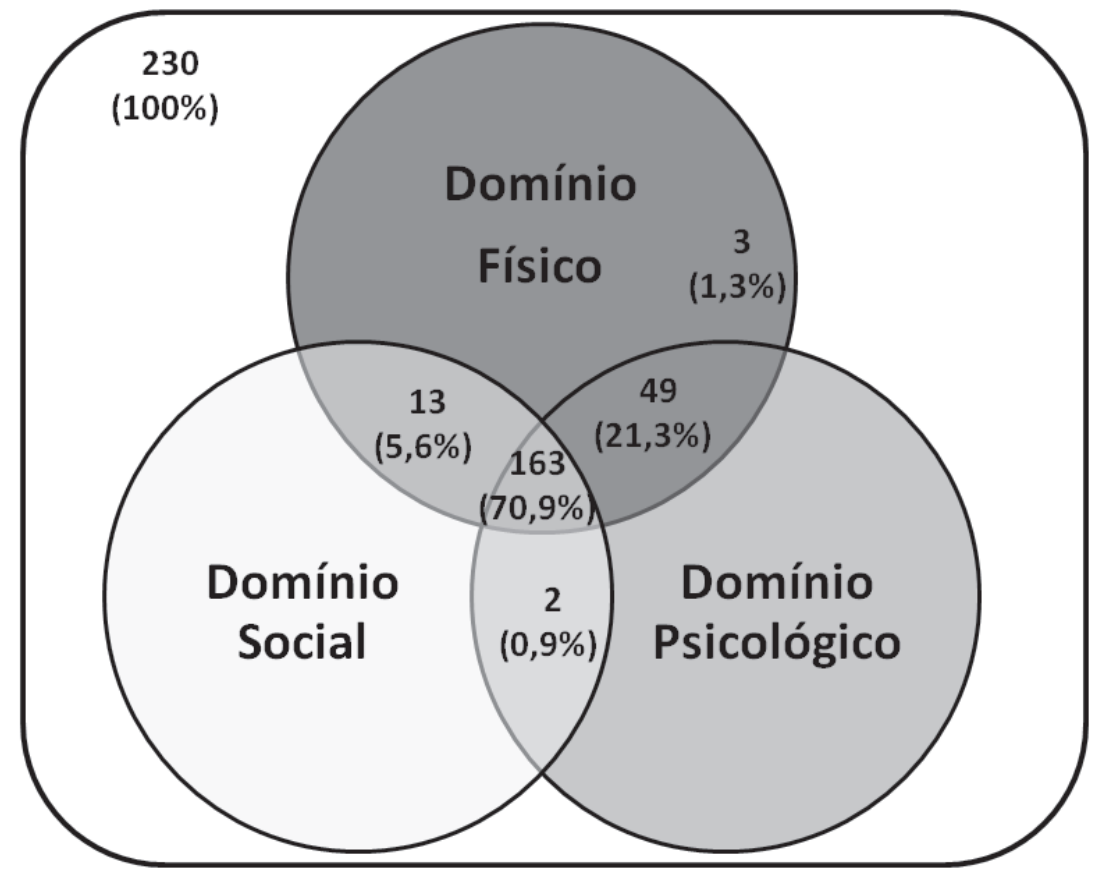

Figura 1. Relação entre os domínios e a prevalência de fragilidade. 
As seguintes variáveis mostraram-se associadas à fragilidade nesta população: idade mais elevada (RP=1,29 IC95\% 1,011,64); analfabetismo ( $\mathrm{RP}=1,31$ IC95\% 1,091,59); comorbidades ( $\mathrm{RP}=1,48$ IC95\% 1,22-
1,79); polifarmácia (utilização de cinco ou mais medicações) ( $R P=1,28$ IC95\% 1,07-1,53); consumo atual de bebida alcoólica $(\mathrm{RP}=0,65$ IC955 0,44-0,95), segundo a tabela 1.

Tabela 1. Prevalência de fragilidade segundo características sociodemográficas, de saúde e de estilo de vida, de idosos de Campo Grande-MS, Cuiabá-MG, Juiz de Fora-MG e Rio de Janeiro-RJ, período 2010-2011.

\begin{tabular}{|c|c|c|c|}
\hline Variáveis & n (442) & Frágil (\%) & RP (IC 95\%) \\
\hline \multicolumn{4}{|l|}{ Sexo } \\
\hline Masculino & 284 & $142(61,7)$ & 1 \\
\hline Feminino & 158 & $88(38,3)$ & $1,11(0,93-1,34)$ \\
\hline \multicolumn{4}{|l|}{ Faixa etária } \\
\hline 60-69 anos & 126 & $55(24,9)$ & 1 \\
\hline 70-79 anos & 158 & $84(38,0)$ & $1,22(0,95-1,56)$ \\
\hline 80 anos ou mais & 146 & $82(37,1)$ & $1,29(1,01-1,64)$ \\
\hline \multicolumn{4}{|l|}{ Escolaridade bivariada } \\
\hline Alfabetizado & 309 & $146(68,5)$ & 1 \\
\hline Não alfabetizado & 108 & $67(31,5)$ & $1,31(1,09-1,59)$ \\
\hline \multicolumn{4}{|l|}{ Estado conjugal } \\
\hline Casado/união estável & 57 & $27(11,8)$ & 1 \\
\hline Divorciado/separado & 66 & $34(14,8)$ & $1,09(0,76-1,56)$ \\
\hline Viúvo & 109 & $62(27,1)$ & $1,20(0,87-1,65)$ \\
\hline Solteiro & 207 & $106(46,3)$ & $1,08(0,80-1,47)$ \\
\hline \multicolumn{4}{|c|}{ Refere duas ou mais morbidades } \\
\hline Não & 209 & $87(38,2)$ & 1 \\
\hline $\operatorname{Sim}$ & 229 & $141(61,8)$ & $1,48(1,22-1,79)$ \\
\hline \multicolumn{4}{|c|}{ Utiliza mais de cinco medicamentos } \\
\hline Não & 309 & $149(64,8)$ & 1 \\
\hline $\operatorname{Sim}$ & 131 & $81(35,2)$ & $1,28(1,07-1,53)$ \\
\hline \multicolumn{4}{|l|}{ Tabagismo } \\
\hline Nunca fumou & 148 & $73(32,0)$ & 1 \\
\hline Tabagismo atual & 148 & $82(36,0)$ & $1,12(0,90-1,40)$ \\
\hline Ex-tabagista & 138 & $73(32,0)$ & $1,07(0,85-1,35)$ \\
\hline \multicolumn{4}{|l|}{ Consumo de bebida alcoólica } \\
\hline Nunca teve o hábito & 145 & $82(35,8)$ & 1 \\
\hline Consome atualmente & 52 & $19(8,3)$ & $0,65(0,44-0,95)$ \\
\hline Não consome mais & 236 & $128(55,9)$ & $0,96(0,80-1,15)$ \\
\hline \multicolumn{4}{|c|}{$\begin{array}{l}\text { Nos últimos } 12 \text { meses realizou atividades para } \\
\text { se distrair, pelo menos, uma vez por semana? }\end{array}$} \\
\hline $\operatorname{Sim}$ & 135 & $64(28,1)$ & 1 \\
\hline Não & 304 & $164(71,9)$ & $1,14(0,93-1,40)$ \\
\hline \multicolumn{4}{|l|}{ Tem religião } \\
\hline $\operatorname{Sim}$ & 407 & $210(92,5)$ & 1 \\
\hline Não & 29 & $17(7,5)$ & $1,14(0,83-1,56)$ \\
\hline
\end{tabular}


$\mathrm{Na}$ análise multivariada, após ajustamento, somente as variáveis escolaridade (não alfabetizados), a presença de comorbidades e o tabagismo atual mantiveram associação estatisticamente significativa com a fragilidade. (tabela 2).

Tabela 2. Análise múltipla pela regressão de Poisson dos fatores associados à fragilidade em idosos brasileiros institucionalizados, Campo Grande-MS, Cuiabá-MG, Juiz de Fora-MG e Rio de Janeiro-RJ, período 2010-2011.

\begin{tabular}{lc}
\hline \multicolumn{1}{c}{ Variável } & Razão de Prevalências ajustadas* (IC de 95\%) \\
\hline Faixa etária & $1,27(1,00-1,61)$ \\
$\quad 70$ anos ou mais & $1,28(1,07-1,54)$ \\
Escolaridade & \\
$\quad$ Não alfabetizados & $1,48(1,21-1,81)$ \\
Comorbidades & \\
$\quad$ Sim & $1,22(1,00-1,47)$ \\
Polifarmácia & \\
$\quad$ Sim & $1,26(1,03-1,53)$ \\
Tabagismo atual & \\
$\quad$ Sim &
\end{tabular}

*Ajustadas por cidade de localização da instituição, sexo e pelas variáveis contidas no modelo.

\section{DISCUSSÃO}

No presente estudo, mais da metade $(52 \%)$ dos idosos avaliados foram considerados frágeis. $\mathrm{Na}$ literatura, foram identificadas somente duas investigações realizadas com idosos institucionalizados, ambas realizadas em países desenvolvidos. ${ }^{19,20}$

A primeira foi realizada no Canadá, com 1.089 indivíduos de 65 anos de idade ou mais, residentes em Assisted Living Facilities, utilizando o instrumento unidimensional Cardiovascular Health Study Scale (CHS scale). A média de idade da população estudada correspondeu a 84,9 $(7,3)$ anos e houve predomínio do sexo feminino $(76,7 \%)$. No estudo, a avaliação inicial efetuada com base na operacionalização das variáveis proposta pelo grupo de Fried et al., ${ }^{4} 48,0 \%$ dos idosos foram considerados frágeis; entretanto, utilizando pontos de corte específicos para a população de estudo, atribuídos a cada item, a prevalência observada foi 19,0\% (Freiheit et al. ${ }^{19}$ ).
$\mathrm{Na}$ outra investigação, realizada com 86 idosos poloneses de nursing homes, com média de idade de 83,8 (8,3) anos e que também apresentava predomínio das mulheres $(76,7 \%)$, foi utilizado o índice de fragilidade Canadian Study of Health and Aging-Clinical Frailty Scale (CSHA-CFS), observando prevalência de fragilidade severa (escore $=7$ ) igual a $34,9 \% \cdot{ }^{20}$

A variação nas estimativas de prevalência se deve, em parte, aos diferentes instrumentos utilizados para avaliação da condição, além das características específicas dos grupos estudados. A população do presente estudo apresentava predomínio de idosos do sexo masculino e média de idade muito inferior àquela identificada nos estudos citados.

Apesar disso, pode-se considerar que as prevalências observadas entre os indivíduos institucionalizados nas investigações citadas são valores relativamente baixos, uma vez que a maioria dos estudos realizados com idosos da 
comunidade em países desenvolvidos aponta a institucionalização como um dos principais desfechos adversos da fragilidade., ${ }^{4,21}$

No Brasil, a institucionalização se associa à maior vulnerabilidade social, à presença de comorbidades e de incapacidade funcional. ${ }^{22}$ Entretanto, da mesma forma, a prevalência de fragilidade observada neste estudo foi inferior ao que se esperaria, com base na associação entre essa condição e a institucionalização, descrita na literatura. ${ }^{4-7}$ À luz destes achados, talvez se faça necessário reavaliar o papel da institucionalização como desfecho adverso da fragilidade em novas investigações.

O único estudo que avaliou a fragilidade por meio do TFI foi realizado com holandeses de 75 anos de idade ou mais da população geral. Nele, a prevalência de fragilidade estimada foi de $47 \%$ e as médias dos escores do TFI foram iguais a: escore total $=4,7$; escore do físico $=2,6$; do domínio psicológico $=0,9$; e do domínio social $=1,2 \cdot{ }^{10}$ Tais resultados são similares aos observados no presente estudo. Os idosos holandeses, embora residentes em comunidade, apresentavam média de idade de $80(3,8)$ anos, superior à identificada nos idosos do presente estudo, o que pode ter contribuído para a similaridade destes resultados.

Como praticamente todos os estudos que analisaram a fragilidade em idosos contemplaram grupos populacionais vivendo na comunidade, a título de comparação, nesta investigação optou-se por avaliar, na medida do possível, as associações entre a fragilidade e as variáveis utilizadas pelos autores do instrumento original. ${ }^{10}$ No estudo holandês, foi efetuada a análise de regressão múltipla hierárquica em duas etapas: na primeira, na qual não foram incluídos "comorbidade", "idade", "sexo feminino" e "estilo de vida não saudável", assim como "renda", mostraramse estatisticamente associados a maior grau de fragilidade; na segunda etapa, com a inclusão da variável "comorbidade", que apresentou associação com "fragilidade", "idade" e "sexo", perderam a significância estatística. Ambos os modelos ainda incluíam as variáveis "nível de escolaridade" e "eventos da vida (morte de alguém querido, adoecimento, entre outros)". ${ }^{10}$
Neste estudo, "faixa etária" e "sexo" não se mostraram associados a "fragilidade", embora a faixa etária tenha alcançado um valor limítrofe, o que pode ser atribuído, em parte, ao tamanho reduzido da população de estudo. Além disso, talvez estas variáveis se tornem menos importantes no contexto da institucionalização no Brasil, uma vez que os fatores que costumam determinar essa prática são a vulnerabilidade social e a condição de saúde, e não o sexo ou a idade.

No presente estudo, como características de estilo de vida não saudável, foram avaliados o consumo de álcool e de tabaco. Foi identificada associação estatisticamente significativa entre o tabagismo atual e a fragilidade, que se manteve no modelo multivariado. Todavia, na análise bivariada, observou-se associação inversa entre o hábito de consumir bebidas alcoólicas e a condição, que embora improvável, já havia sido relatada na literatura entre chineses de 70 anos de idade ou mais. ${ }^{23}$ É comum que indivíduos doentes tendam a abster-se de hábitos deletérios, sugerindo que os idosos mais saudáveis ainda consumiriam bebidas alcoólicas, explicando sua menor probabilidade de serem frágeis. ${ }^{23} \mathrm{Na}$ maioria das instituições de longa permanência para idosos na qual este estudo foi realizado, os indivíduos sem déficit cognitivo e funcionalmente independentes podem deixar as instituições por períodos de tempo determinados, durante o dia, o que possibilitaria seu acesso ao álcool e tabaco observados nesta investigação.

No estudo holandês, não foi observada associação entre o nível de escolaridade e a fragilidade. ${ }^{10}$ Neste estudo, o analfabetismo mostrou associação positiva com a condição, inclusive na análise multivariada. Acreditase que essa diferença se deva às diferenças socioculturais entre os dois países. Devido ao baixo nível de escolaridade de grande parcela da população do estudo brasileiro, optou-se por recategorizar os indivíduos em "alfabetizados" e "não alfabetizados". Na Holanda, mais de 60\% da população tinha, pelo menos, nível médio de escolaridade, talvez por isso o analfabetismo foi avaliado conjuntamente com o nível primário de educação. 
As comorbidades, representadas pela referência a duas ou mais morbidades, também se apresentaram como fator de risco para a fragilidade, e esta variável permaneceu com significância estatística após ajustamento pelas demais. Utilizando outro instrumento de avaliação, o CHS Scale, a presença de duas ou mais comorbidades aumentou 2,68 vezes a probabilidade de apresentar fragilidade em idosos brasileiros do estudo Salud Bienestar y Envejecimiento (SABE), ${ }^{24}$ e para cada morbidade dos idosos espanhóis da coorte Peñagrande, essa probabilidade aumentava $27 \% .{ }^{25}$

Neste estudo, o estado conjugal viúvo/ solteiro foi associado à fragilidade, embora a significância estatística não tenha se mantido no modelo multivariado. Essa associação já havia sido observada em outras investigações, como no estudo SABE realizado no Brasil, no qual entre os indivíduos que não tinham companheiro, observou-se maior risco de fragilidade em relação aos que tinham $(1,32 \mathrm{IC}=1,08-1,62) \cdot{ }^{24}$ Entre taiwaneses de 65 anos de idade ou mais, além de sexo feminino e idades mais avançadas, características como menor escolaridade e estado conjugal solteiro/viúvo apresentaram-se como fatores de risco para a fragilidade. ${ }^{26}$

Foi observada maior prevalência de fragilidade entre os idosos que faziam uso de polifarmácia, representada pelo uso de mais de cinco medicações; entretanto, essa associação não se manteve na análise multivariada. $\mathrm{O}$ uso de múltiplas medicações reflete, em parte, a condição de saúde geral do indivíduo, especialmente no que concerne ao número de comorbidades que apresenta. $^{27}$

Entre as limitações deste estudo, encontra-se seu desenho seccional, que não permite avaliar a sequência temporal entre a exposição e o desfecho. Outra questão se refere ao número de participantes do estudo, o que pode ter contribuído para a impossibilidade de se obter associações com significância estatística entre a fragilidade e algumas covariáveis analisadas.

Este estudo é uma das poucas investigações a utilizar um instrumento de avaliação de fragilidade validado para a cultura dos pesquisados. Constituiu, também, a primeira abordagem do tema "fragilidade em idosos institucionalizados na América Latina". É preciso ressaltar, no entanto, que a validação da versão brasileira do instrumento foi realizada com idosos que vivem em comunidade e não em residentes de ILPS.

Acredita-se que a avaliação da fragilidade, com vistas à intervenção precoce, deve ser uma prioridade na atenção primária à saúde. ${ }^{2,28}$ Conhecer os fatores associados a esta condição em agrupamentos de idosos com características particulares é de fundamental importância na saúde pública. Em estudo recente, realizado na Holanda, este instrumento se mostrou preditivo para indicadores de utilização de serviços de saúde em um e dois anos de seguimento.

É necessário realizar estudos que utilizem o TFI para investigar a prevalência de fragilidade e os fatores associados a essa condição em indivíduos idosos residentes na comunidade. Da mesma forma, é também importante avaliar a validade preditiva deste instrumento quanto aos desfechos adversos de saúde associados à fragilidade na população idosa brasileira.

\section{CONCLUSÕES}

A prevalência de fragilidade na população de estudo foi relativamente alta e superou os valores observados nos outros dois estudos que avaliaram esta condição em idosos institucionalizados. Esperava-se, entretanto, encontrar prevalência ainda mais alta, dado que a institucionalização é apontada na literatura como um dos desfechos adversos da fragilidade. Diante desses resultados, considera-se importante realizar estudos que reavaliem o papel desta condição na predição de institucionalização.

As associações identificadas são corroboradas pela literatura científica, em avaliações com idosos residentes em comunidade, e as variações observadas podem ser atribuídas aos diferentes instrumentos utilizados na avaliação e às características distintas dos grupos estudados. Nos estudos sobre fragilidade em idosos 
institucionalizados, não foram avaliados fatores associados a essa condição.

Este estudo utilizou um instrumento multidimensional de avaliação da fragilidade validado para a população brasileira, sendo observadas boa compreensão e aceitabilidade por parte dos idosos institucionalizados. É importante realizar outros estudos que avaliem tanto a utilização do Tilburg Frailty Indicator (TFI) em idosos residentes em comunidade, quanto a validade preditiva do instrumento na população brasileira.

\section{REFERÊNCIAS}

1. De Vries NM, Staal JB, Van Ravensberg CD, Hobbelen JSM, Olde Rikkert MGMO, Nijhuisvan der Sanden MWG. Outcome instruments to measure frailty: a systematic review. Ageing Res Rev 2011;10(1):104-14.

2. Pialoux T, Goyard J, Lesourd B. Screening tools for frailty in primary health care: a systematic review. Geriatr Gerontol Int 2012;12(2):189-97.

3. Gobbens RJJ, Luijkx KG, Wijnen-Sponselee MT, Schols JMGA. In search of an integral conceptual definition of frailty: opinions of experts. J Am Med Dir Assoc 2010;11(5):338-43.

4. Fried LP, Tangen CM, Walston J, Newman AB, Hirsch C, Gottdiener J, et al. Frailty in older adults: evidence for a phenotype. J Gerontol Ser A Biol Sci Med Sci 2001;56(3):146-56.

5. Ensrud KE, Ewing SK, Taylor BC, Fink HA, Cawthon PM, Stone KL et al. Comparison of 2 frailty indexes for prediction of falls, disability, fractures, and death in older women. Arch Intern Med 2008;168(4):382-9.

6. Berges I, Graham JE, Ostir GV, Markides KS, Ottenbacher KJ. Sex Differences in Mortality among Older Frail Mexican Americans. J Womens Health 2009;18(10):1647-51.

7. Gobbens RJJ, Van Assen MALM. Frailty and its prediction of disability and health care utilization: the added value of interviews and physical measures following a self-report questionnaire. Arch Gerontol Geriatr 2012;55(2):369-79.

8. Frieswijk N, Buunk BP, Steverink N, Slaets JPJ. The interpretation of social comparison and its relation to life satisfaction among elderly people: does frailty make a difference? J Gerontol Ser B Psychol Sci Soc Sci 2004;59B(5):250-7.

9. Folsom AR, Boland LL, Cushman M, Heckbert SR, Rosamond WD, Walston JD. Frailty and risk of venous thromboembolism in older adults. J Gerontol Ser A Biol Sci Med Sci 2007;62(1):79-82.

10. Gobbens RJJ, Van Assen MALM, Luijkx KG, Wijnen-Sponselee MT, Schols JMGA. Determinants of Frailty. J Am Med Dir Assoc 2010;11(5):356-64.

11. Rothman MD, Leo-Summers L, Gill TM. Prognostic significance of potential frailty criteria. J Am Geriatr Soc 2008;56(12):2211-6.

12. Gobbens RJJ, Van Assen MALM, Luijkx KG, Wijnen-Sponselee MT, Schols JMGA. The Tilburg Frailty Indicator: psychometrics properties. J Am Med Dir Assoc 2010;11(5):344-55.

13. Santiago LM, Luz LL, Mattos IE, Gobbens RJJ. Adaptação transcultural do instrumento Tilburg Frailty Indicator (TFI) para a população brasileira. Cad Saúde Pública 2012;28(9):1795-1801.

14. Santiago LM, Luz LL, Mattos IE, Gobbens RJJ, Van Assen MALM (TFI). Psychometric properties of the Brazilian version of the Tilburg Frailty Indicator. Arch Gerontol Geriatr 2013;57(1):39-45

15. Brasil. Ministério da Saúde. Estatuto do idoso. Brasília (DF): Ministério da Saúde; 2003.

16. Brasil. Instituto de Pesquisa Econômica Aplicada. Características das instituições de longa permanência para idosos - região Sudeste. Ana Amélia Camarano, Coordenadora. Brasília (DF): IPEA; 2010.

17. BRASIL. Portaria n. 2874 de 30 de agosto de 2000. Altera dispositivos da portaria SEAS 2854/2000. Diário Oficial da República Federativa do Brasil (DF), 04 set 2000; Seção 1:171-E.

18. Rockwood K, Abeysundera MJ, Mitnitski A. How should we grade frailty in nursing home patients? J Am Med Dir Assoc 2007;8(9):595-603.

19. Freiheit EA, Hogan DB, Strain LA, Schmaltz HN, Patten SB, Eliasziw M, et al. Operationalizing frailty among older residents of assisted living facilities. BMC Geriatr 2011;11:23.

20. Matusik P, Tomaszewski K, Chmielowska K, Nowak J, Nowak W, Parnicka A, et al. Severe frailty and cognitive impairment are related to higher mortality in 12-month follow-up of nursing home residents. Arch Gerontol Geriatr 2012;55(1):22-4. 
21. Rockwood K, Stadnyk K, MacKnight C, McDowell I, Herbert R, Hogan DB. A brief clinical instrument to classify frailty in elderly people. Lancet 1999; 353(9148):202-06.

22. Lopes FL, Tier CG, Lunardi Filho W, Santos SSC. Diagnóstico de enfermagem de idosos residentes em uma instituição de longa permanência (ILP). Ciênc Cuid Saude 2007;6(1):59-67.

23. Woo J, Goggins W, Sham A, Ho SC. Social Determinants of Frailty. Gerontology 2005;51(6):402-8.

24. Alvarado BE, Zunzunegui MV, Béland F, Bamvita JM. Life course social and health conditions linked to frailty in Latin American older men and women. J Gerontol Ser A Biol Sci Med Sci 2008;63(12):1399-1406.
25. Alcalá MVC, Puime AO, Santos MTS, Barral AG, Montalvo JIG, Zunzunegui MV. Prevalence of frailty in an elderly Spanish urban population. Relationship with comorbidity and disability. Aten Primaria 2010;42(10):520-7.

26. Chen CY, Wu SC, Chen LJ, Lue BH. The prevalence of subjective frailty and factors associated with frailty in Taiwan. Arch Gerontol Geriatr 2010;50 Suppl 1:S43-7.

27. Luccheti G, Granero AL, Pires SL, Gorzoni ML. Fatores associados à polifarmácia em idosos institucionalizados. Rev Bras Geriatr Gerontol 2010;13(1):51-8.

28. Strandberg TE, Pitkälä KH. Frailty in elderly people. Lancet 2007;369(9570):1328-9. 